\title{
Analysis of Microwave Sintering of Ceramics
}

\author{
Anindita Chatterjee, Tanmay Basak, and K. G. Ayappa \\ Dept. of Chemical Engineering, Indian Institute of Science, Bangalore 560012, India
}

\begin{abstract}
The microwave heating of ceramic materials has been analyzed by solving the equations for grain growth and porosity (Svoboda and Riedel, 1992) during the late stages of sintering, coupled with the heat conduction equation and electric field equations for $1-D$ slabs. Microwave power absorption and heating profiles have been calculated for $\mathrm{Al}_{2} \mathrm{O}_{3}$ and $\mathrm{SiC}$ in the absence of sintering, and calculations have been carried out to study the effect of increasing dielectric loss of $\mathrm{Al}_{2} \mathrm{O}_{3}$ as a function of temperature. A comparison of the densification and grain growth for $\mathrm{Al}_{2} \mathrm{O}_{3}$ during microwave and conventional sintering indicates that within the framework of the present model, there is no difference between the two heating modes during the late stages of sintering.
\end{abstract}

\section{Introduction}

Microwaves (MWs) are electromagnetic waves with frequencies ranging from $300 \mathrm{MHz}$ to $300 \mathrm{GHz}$, and wavelengths ranging from a few $\mathrm{cm}$ to a few $\mathrm{mm}$. Unlike conventional heating, the penetration of microwaves into materials gives rise to a volumetrically distributed heat source. Consequently, microwave processing makes it possible to rapidly heat both small and large samples with greater uniformity when compared with conventional heating. Although the presence of water molecules allows food materials to rapidly heat when exposed to MWs, the ability of many ceramics to absorb MW radiation above a critical temperature has led to the use of microwaves for processing of ceramics during drying, clinkering, sintering, melting and joining (Sutton, 1989). Synthesis of a variety of materials via MW processing has been discussed by Rao and Ramesh (1995). The ceramics that are commonly sintered using microwaves are $\mathrm{Al}_{2} \mathrm{O}_{3}, \mathrm{ZrO}_{2}$, $\mathrm{MgO}$, and $\mathrm{SiC}$. In addition to shorter processing times, microwave sintering results in ceramics with improved microstructure and mechanical properties.

The most intriguing effects observed during MW sintering are the higher densification (Janney and Kimrey, 1988; Samuels and Brandon, 1992; Xie et al., 1996), and reaction rates (Rao and Ramesh, 1995) when compared with conventioned sintering. It has been observed that microwave heating densifies $\mathrm{Al}_{2} \mathrm{O}_{3}$ far more rapidly than conventional heating, and at lower temperatures densification is higher for microwave heating when compared with conventional heating. The reasons for the higher densification rates have been attributed to a lower activation energy for MW sintering (Jan-

Correspondence concerning this article should be addressed to K. G. Ayappa. ney and Kimrey, 1988; Samuels and Brandon, 1992). Although a specific model or mechanism is yet to be developed, the higher densification observed during MW sintering is generally thought to be due to enhanced grain boundary diffusion. A heat-transfer calculation by Johnson (1991) of a planar model indicates that the temperature difference between grain boundaries and grains is less than a degree illustrating that the influence of MWs is not merely a thermal effect. Rothman (1994) reviews the experimental literature on diffusion coefficient measurements in the presence of a MW field.

During microwave heating electrical energy from the microwaves is converted into heat. The MW power absorbed by a sample is proportional to the electric field distribution in the sample, which is determined by the electrical properties of the material. The electric field distribution and the absorbed MW power can be computed by solving Maxwell's equations. With a knowledge of the absorbed MW power, the temperature distribution in a sample is obtained by solving the appropriate energy balance equations. Using this continuum approach, models for MW-based transport processes like heating, drying, and thawing in dielectric materials exposed to plane electromagnetic waves have been developed. The literature has been recently reviewed by Ayappa (1997).

Although a large body of experimental literature exists on MW and hybrid sintering of various ceramics, efforts to model the process have been relatively few. An asymptotic analysis for the heating of a semi-infinite ceramic slab exposed to a plane wave has been carried out by Kriegsmann et al. (1990). Meek (1987) proposes a qualitative model for MW heating of a porous ceramic based on the equations for the electric field 
at a lossless dielectric interface. Using a heat conduction model, Thomas et al. (1994) study the heating of alumina cylinders with a temperature-dependent source term.

During $M W$ sintering, in addition to heating, the rise in temperature is accompanied by an increase in grain diameter and a consequent decrease in the porosity during densification. In order to consistently model the sintering phenomenon, equations that describe the evolution of porosity and grain diameter must be coupled with the electric field and energy balance equations. Although evolution equations for the porosity and grain growth during the initial and intermediate stages of sintering have yet to be developed, continuum equations for the evolution of the grain diameter and porosity during the late stages of sintering (densification greater than 90\%) have been developed by Svoboda and Riedel (1992). To our knowledge, models that combine Maxwell's equations with evolution equations for grain growth and porosity of a sample during microwave sintering have yet to appear in the literature.

One of the main objectives of the present work is to consistently solve Maxwell's equations, along with the equations for heat transfer, grain growth, and porosity and compare the densification and grain growth predicted during microwave sintering with those predicted by conventional sintering. The basic equations for the electric field, temperature and evolution equations for the grain diameter and porosity are developed. The power absorption and heating in the absence of sintering between slabs of $\mathrm{Al}_{2} \mathrm{O}_{3}$ and $\mathrm{SiC}$ are contrasted, followed by heating of $\mathrm{Al}_{2} \mathrm{O}_{3}$ with a temperature-dependent dielectric loss. Next, we model the $\mathrm{MW}$ sintering of $\mathrm{Al}_{2} \mathrm{O}_{3}$ by solving the evolution equations for grain diameter and porosity during the late stages of sintering. Using the heating rates observed during MW heating, the grain growth and porosity obtained for conventional sintering are contrasted with those observed in the MW heating model.

\section{Theory}

\section{Electric field equations}

We are interested in the sintering of a 1-D slab exposed to a plane electromagnetic wave as shown in Figure 1. For plane waves, the electric and magnetic components lie in a plane $(x-y)$ of uniform intensity and vary only in the direction of propagation $(Z)$. For this case, the equation for electric field $E_{x}$ is

$$
\frac{d^{2} E_{x}}{d Z^{2}}+k^{2}(\phi) E_{x}=0
$$

where $k=\omega / c \sqrt{\kappa^{\prime}(\phi)+i \kappa^{\prime \prime}(\phi)}$ is the propagation constant dependent on $\kappa^{\prime}$, the relative dielectric constant and $\kappa^{\prime \prime}$, the relative dielectric loss. Here $\omega=2 \pi f$, where $f$ is the frequency of the electromagnetic radiation and $c$ is the velocity of light. Since the porosity, $\phi$ of the material changes as the material is being sintered, $\kappa^{\prime}$ and $\kappa^{\prime \prime}$ are functions of $\phi(T)$. Equation 1 is derived from Maxwell's curl equations assuming time-harmonic electric and magnetic fields in an electroneutral medium where the time scale for diffusive transport is much larger than the time scale for electromagnetic wave propagation (Ayappa, 1997). We restrict our analysis to

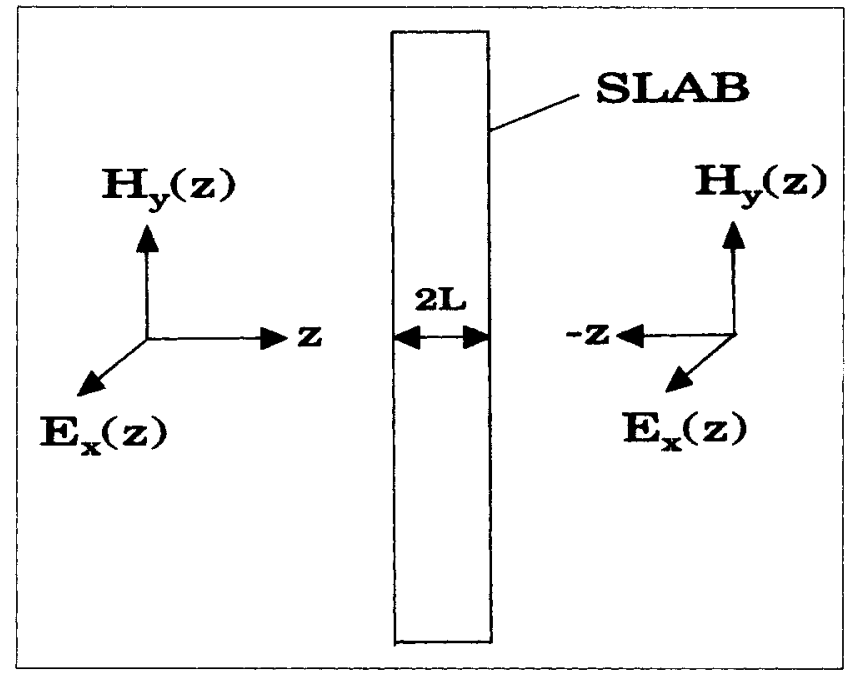

Figure 1. Slab exposed to plane waves.

nonmagnetic ceramics such as $\mathrm{Al}_{2} \mathrm{O}_{3}$ and $\mathrm{SiC}$, whose magnetic permeability is well approximated by the free space value. However there are some ceramics like ferrites in which magnetic effects cannot be neglected.

We seek solutions to Eq. 1 for a slab exposed to plane waves at a fixed intensity and frequency that are incident normally on the face of the sample as shown in Figure 1. For a slab exposed to radiation of intensity $E_{L}$ from the left as shown in Figure 1, the boundary condition (Ayappa et al., 1991) at the face $Z=-L$ is

$$
\frac{d E_{x}}{d Z}+\frac{i \omega E_{x}}{c}=\frac{2 i \omega}{c} E_{L} e^{-i \omega L / c}
$$

Similarly, the boundary condition at the face $Z=L$ exposed to radiation of intensity $E_{R}$ is

$$
\frac{d E_{x}}{d Z}-\frac{i \omega E_{x}}{c}=-\frac{2 i \omega}{c} E_{R} e^{-i \omega L / c}
$$

With a knowledge of the electric field intensity in the medium, the local power dissipated is

$$
p(Z)=\frac{1}{2} \omega \epsilon_{0} \kappa^{\prime \prime} E_{x}^{2}
$$

where $\epsilon_{0}$ is the free-space permittivity.

A ceramic being sintered is essentially a porous medium whose porosity decreases as sintering proceeds. Since we are interested only in the late stages of sintering, we assume the solid to form the continuous phase. The effective dielectric properties of the porous medium at a given porosity $\phi$ are calculated using the Maxwell-Wagner mixture rule

$$
\kappa^{*}=\frac{\kappa_{s}^{*}\left[\kappa_{a}^{*}(1+2 \phi)+2 \kappa_{s}^{*}(1-\phi)\right]}{\kappa_{a}^{*}(1-\phi)+\kappa_{s}^{*}(2+\phi)},
$$


where $\kappa^{*}=\kappa^{\prime}+i \kappa^{\prime \prime}$ is the relative complex dielectric constant for the porous media and $\kappa_{a}^{*}$ and $\kappa_{s}^{*}$ are the relative complex dielectric constants of air and solid phases, respec tively.

\section{Microwave heating in a slab}

Consider a slab of thickness $2 L$ exposed to microwaves, as shown in Figure 1. The surrounding medium is at an ambient temperature during the entire heat-up period, and heat is lost by convection and radiation at the sample boundaries. Assuming local thermal equilibrium between the ceramic matrix and air at the pore scale, the energy balance equation for the ceramics sample exposed to microwaves is

$$
\left(\rho C_{p}\right)_{\mathrm{eff}} \frac{\partial T}{\partial t}=\frac{\partial}{\partial Z}\left(k_{\mathrm{eff}} \frac{\partial T}{\partial Z}\right)+p(T(Z)),
$$

where $\rho$ is the density, $C_{p}$ is the specific heat capacity, $T$ is the temperature and $p(T(Z))$ is a volumetric source term due to microwave power absorption. Equation 6 is solved to obtain transient temperature profiles for a sample exposed to microwave radiation. The effective thermal conductivity

$$
k_{\mathrm{eff}}=(1-\phi) k_{s}+\phi k_{a}
$$

where $k_{s}$ and $k_{a}$ are the thermal conductivities of the solid and air phases, respectively. Similarly, the effective specific heat capacity is

$$
\left(\rho C_{p}\right)_{\mathrm{eff}}=(1-\phi)\left(\rho C_{p}\right)_{s}+\phi\left(\rho C_{p}\right)_{a},
$$

where $\left(\rho C_{p}\right)_{\mathrm{eff}}$ is the effective heat capacity per unit volume.

\section{Evolution equations for porosity and grain diameter during sintering}

Sintering of a powder compact occurs in three stages. The first stage is characterized by neck formation between the particles, the second is characterized by the shrinkage of open porosity, and the third stage is where grain boundary diffusion dominates, and the shrinkage of closed pores occurs (Kingery et al., 1991). All three stages can be accompanied by grain coarsening, but this is most pronounced in the final stages.

Svoboda and Riedel (1992) have developed coupled equations which describe the evolution of the grain size and porosity with time for the late stages of sintering. Since the model is developed for the late stages of sintering, the pores which are situated on the grain boundary facets are assumed to shrink only by grain boundary diffusion. Further, only pores that are attached to the grain boundary can undergo a reduction in porosity. Here, we present only the final forms of the equations. The evolution equation for porosity $\phi$ is

$$
\frac{d \phi}{d t}=\frac{24 \bar{Z} \Omega \delta D_{b}}{k T q(\bar{\omega}) G^{3}}\left[\sigma_{h}-\frac{2 \gamma_{s} \sin \psi}{G}\left(\frac{4 \bar{Z} h(\psi)}{\phi}\right)^{1 / 3}\right] .
$$

Here $\delta D_{b}$ is the grain boundary diffusion coefficient, $q(\vec{\omega})=$ $-2 \ln \bar{\omega}-(3-\bar{\omega})(1-\bar{\omega}), \quad w=4(\phi / 4 \mathrm{~h}(\psi) \bar{z})^{2 / 3} / \beta_{1}^{2}$, where $h(\psi)=\left[(1+\cos \psi)^{-1}-0.5 \cos \psi\right] / \sin \psi \cdot \gamma_{s}$ is the surface en- ergy per unit area, $G$ is the diameter of the grain, $\bar{\omega}$ is the area fraction of grain boundary occupied by pores, $k$ is the Boltzmann's constant, $T$ is the temperature, $\psi$ is the dihedral angle, $\Omega$ is the atomic volume, and $\bar{Z}$ is the number of neighbors of the central grain. In deriving Eq. 9 the normal stress on the individual boundaries was replaced by the hydrostatic stress $\sigma_{h}$ in order to average over the orientations of the boundary facets. The evolution equation for the grain diameter $G$ is

$$
\frac{d G}{d t}=\frac{\alpha_{1} M_{b} \gamma_{b}}{G}\left[1-\bar{\omega}+\frac{0.42 \eta M_{b} k T G^{2}}{\beta_{1}^{2} p(\psi) \Omega \delta D_{s}}\left(\frac{\phi}{\bar{Z} h(\psi)}\right)^{4 / 3}\right]^{-1},
$$

where $\alpha_{1}=1$ for three-dimensional (3-D) grain structures, $M_{b}$ is the grain boundary mobility, $\gamma_{b}$ is the grain boundary energy per unit area, $\eta$ is a factor which depends on the shape of the migrating grain boundary, $\beta_{1}$ is a factor determined from the condition that the surface area of the $\bar{Z}$ grain facets is equal to the surface area of the sphere, $\delta D_{s}$ is the surface diffusion coefficient expressed in terms of the grain boundary thickness $\delta$ and $p(\psi)=2(1+\cos \psi)^{2} /(2+\cos \psi)$. Equations 9 and 10 represent coupled evolution equations for $\phi$ and $G$.

\section{Dimensionless forms}

If the intensity of the electric field incident from the left is $E_{L}\left(\mathrm{~V} \cdot \mathrm{m}^{-1}\right)$, then defining the dimensionless variables

$$
v_{x}+i w_{x}=\frac{E_{x}}{E_{L}} \quad \text { and } \quad z=\frac{Z+L}{2 L}
$$

the equation for the electric fields (Eq. 1) obtained by equating the real $\left(v_{x}\right)$ and imaginary $\left(w_{x}\right)$ components are

$$
\frac{d^{2} v_{x}}{d z^{2}}+\psi v_{x}-\chi w_{x}=0
$$

and

$$
\frac{d^{2} w_{x}}{d z^{2}}+\chi v_{x}+\psi w_{x}=0
$$

where $\psi=\left(4 L^{2} \omega^{2} / c^{2}\right) \kappa^{\prime}(\phi)$ and $\chi=\left(4 L^{2} \omega^{2} / c^{2}\right) \kappa^{\prime \prime}(\phi)$. The boundary conditions for the real and imaginary components from Eqs. 2 and 3 are

$$
\left.\begin{array}{l}
\frac{d v_{x}}{d z}-\frac{2 \omega L}{c} w_{x}=\frac{4 \omega L}{c} \sin \left(\frac{\omega L}{c}\right) \\
\frac{d w_{x}}{d z}+\frac{2 \omega L}{c} v_{x}=\frac{4 \omega L}{c} \cos \left(\frac{\omega L}{c}\right)
\end{array}\right\} \text { at } z=0
$$

and

$$
\left.\begin{array}{l}
\frac{d v_{x}}{d z}+\frac{2 \omega L}{c} w_{x}=-\frac{E_{R}}{E_{L}} \frac{4 \omega L}{c} \sin \left(\frac{\omega L}{c}\right) \\
\frac{d w_{x}}{d z}-\frac{2 \omega L}{c} v_{x}=-\frac{E_{R}}{E_{L}} \frac{4 \omega L}{c} \cos \left(\frac{\omega L}{c}\right)
\end{array}\right\} \text { at } z=1
$$


Using $\rho_{0}, C_{p, 0}$ and $k_{0}$ as the reference thermal properties, the transient 1-D heat conduction equation for a slab (Eq. 6) is

$$
\overline{\rho C_{p}} \frac{\partial \theta}{\partial \tau_{1}}=\frac{\partial}{\partial z}\left(\bar{k} \frac{\partial \theta}{\partial z}\right)+P(z(\theta))
$$

where

$$
z=\frac{Z+L}{2 L}, \quad \theta=\frac{T-T_{\infty}}{T_{0}}, \quad \tau_{1}=\frac{\alpha_{0} t}{4 L^{2}}
$$

and

$$
P=\frac{4 p L^{2}}{k_{0} T_{0}}, \quad \overline{\rho C_{p}}=\frac{\left(\rho C_{p}\right)_{\mathrm{eff}}}{\rho_{0} C_{p, 0}}, \quad \bar{k}=\frac{k_{\mathrm{eff}}}{k_{0}} .
$$

The expression for the microwave power term in Eq. 15 is

$$
P(z, \phi)=\frac{2 L^{2} \omega \epsilon_{0} \kappa^{\prime \prime}(\phi) E_{L}^{2}}{k_{0} T_{0}}\left(v_{x}^{2}+w_{x}^{2}\right)
$$

The boundary conditions are

$$
\frac{\partial \theta}{\partial z}-B i_{c} \theta-B i_{r} F(\theta)=0 \quad \text { at } z=0
$$

and

$$
\frac{\partial \theta}{\partial z}+B i_{c} \theta+B i_{r} F(\theta)=0 \quad \text { at } z=1,
$$

where

$$
\begin{gathered}
B i_{c}=\frac{2 h L}{k_{\text {eff }}}, \quad B i_{r}=\frac{2 \epsilon \sigma T_{\infty}^{3} L}{k_{\text {eff }}} \\
F(\theta)=\left(\frac{T_{0}}{T_{\infty}}\right)^{3} \theta^{4}+4\left(\frac{T_{0}}{T_{\infty}}\right)^{2} \theta^{3}+6\left(\frac{T_{0}}{T_{\infty}}\right) \theta^{2}+4 \theta
\end{gathered}
$$

and initial condition

$$
\theta\left(\tau_{1}=0\right)=\frac{T_{0}-T_{\infty}}{T_{0}} \quad \text { for } 0 \leq z \leq 1
$$

$h$ is the convection heat-transfer coefficient, $\epsilon$ is the emissivity, $\sigma=5.67 \times 10^{-8} \mathrm{~W} \cdot \mathrm{m}^{-2} \mathrm{~K}^{-4}$ is the Stefan-Boltzmann constant, and $T_{0}$ is the initial temperature $(\mathrm{K})$ of the material.

The evolution equations (Eqs. 9 and 10) can be written in dimensionless form if length and time are normalized with $x_{0}$ and $t_{0}$ where

$$
x_{0}=\left(\frac{\gamma_{s} \Omega \delta D_{s}}{\gamma_{b} M_{b} k T_{0}}\right)^{1 / 2} \quad \text { and } \quad t_{0}=\frac{\gamma_{s} \Omega \delta D_{s}}{2\left(\gamma_{b} M_{b}\right)^{2} k T_{0}}
$$

If $\bar{G}=G / x_{0}$ and $\tau_{2}=t / t_{0}$, Eqs. 9 and 10 reduce to

$$
\begin{aligned}
\frac{d \phi}{d \tau_{2}} & =\frac{24 \bar{Z} \Omega^{2} \gamma_{s} \delta D_{s} \delta D_{b}}{2\left(\gamma_{b} M_{b}\right)^{2} k^{2} T_{0} T q(\bar{\omega})\left(\bar{G} x_{0}\right)^{3}} \\
& \times\left[\sigma_{h}-\frac{2 \gamma_{s} \sin \psi}{\bar{G} x_{0}}\left(\frac{4 \bar{Z} h(\psi)}{\phi}\right)^{1 / 3}\right],
\end{aligned}
$$

and

$$
\frac{d \bar{G}}{d \tau_{2}}=\frac{\alpha_{1}}{2 \bar{G}}\left[1-\bar{\omega}+\frac{0.42 \eta M_{b} k T\left(\bar{G} x_{0}\right)^{2}}{\beta_{1}^{2} p(\psi) \Omega \delta D_{s}}\left(\frac{\phi}{\bar{Z} h(\psi)}\right)^{4 / 3}\right]^{-1} .
$$

\section{Results and Discussion}

\section{Numerical analysis}

The Galerkin finite-element method is used to solve the coupled nonlinear Eqs. 11, 12, 15, 20, and 21. An implicit backward difference is used to discretize the time domain and the resulting nonlinear equations are solved using the Newton-Raphson method. The finite-element residual equations are given in the Appendix. The dimensionless time step for the energy balance equations is different from that of the evolution equations for porosity and grain growth, since the parameters used for the dimensionless equations were different in the two cases. Initially, the evolution equations for porosity and grain diameter were solved by the Runge-Kutta method. A dimensionless time step of $\Delta \tau_{2}=0.0125$ was taken for solving the evolution equations for porosity and grain diameter. This value of the dimensionless time step corresponds to a real time step of $0.1119 \mathrm{~s}$, chosen after convergence tests. For the finite-element analysis, 20 quadratic basis functions with three nodes per element and 20 elements were used.

\section{Microwave heating of ceramics}

Preliminary studies were carried out to analyze the microwave heating characteristics of $\mathrm{Al}_{2} \mathrm{O}_{3}$ and $\mathrm{SiC}$ in the absence of sintering. The heat conduction equation (Eq. 15) was solved with the electric field equations (Eqs. 11 and 12) assuming constant material properties. Thermal properties of ceramics have a small dependence on temperature; however, the dielectric loss of most ceramics is a strong function of temperature (Sutton, 1989). We investigate the influence of temperature-dependent dielectric properties in the next section. The dielectric and thermal properties of $\mathrm{Al}_{2} \mathrm{O}_{3}$ and $\mathrm{SiC}$ are tabulated in Table 1 . In all our calculations we assume a convection heat-transfer coefficient, $h=2 \mathrm{~W} \cdot \mathrm{m}^{-2} \cdot \mathrm{K}^{-1}$ which results in the Biot number for convection $\left(B i_{c}\right)$ ranging between $0.0005-0.004$ and an emissivity $\epsilon=0.15$ for both $\mathrm{Al}_{2} \mathrm{O}_{3}$ and $\mathrm{SiC}$ which results in the Biot number for radiation $\left(B i_{r}\right)$ ranging between $0.0006-0.0004$. Results are obtained for microwaves of equal intensity on both faces of the slab.

Figure 2 illustrates the power distribution and evolution of temperature profiles for $\mathrm{Al}_{2} \mathrm{O}_{3}$ and $\mathrm{SiC}$ slabs of thickness 2 
Table 1. Thermal and Dielectric Properties for $\mathrm{Al}_{2} \mathrm{O}_{3}$, $\mathrm{SiC}$ and $\mathrm{Air}^{*}$

\begin{tabular}{lccc}
\hline \multicolumn{1}{c}{ Material Property } & $\mathrm{Al}_{2} \mathrm{O}_{3}$ & $\mathrm{SiC}$ & Air \\
\hline Density, $\rho\left[\mathrm{kg} \cdot \mathrm{m}^{-3}\right]$ & 3,750 & 3,100 & 0.325 \\
$\begin{array}{l}\text { Heat capacity, } C_{p}\left[\mathrm{~J} \cdot \mathrm{kg}^{-1} \cdot \mathrm{K}^{-1}\right] \\
\text { Thermal conductivity, } \mathrm{k} \\
{\left[\mathrm{W} \cdot \mathrm{m}^{-1} \mathrm{~K}^{-1}\right]}\end{array}$ & 1,046 & 3,300 & 1158.93 \\
$\begin{array}{c}\text { Dielectric constant } \\
(2,450 \mathrm{MHz}), \kappa^{\prime}\end{array}$ & 10.8 & 40 & 0.0706 \\
Dielectric loss $(2,450 \mathrm{MHz}), \kappa^{\prime \prime}$ & 0.1566 & 26.66 & 1.0 \\
\hline
\end{tabular}

* Dielectric and thermal properties of $\mathrm{Al}_{2} \mathrm{O}_{3}$ and $\mathrm{SiC}$ obtained from Ceramic Source, Vol, 7, 1991. Thermal properties of air are obtained from Welty et al. (1976).

$\mathrm{cm}$. An initial condition of $700 \mathrm{~K}$ is assumed for the simulation studies with an ambient temperature of $300 \mathrm{~K}$. The wavelength of radiation $\left(\lambda_{m}\right)$ in $\mathrm{Al}_{2} \mathrm{O}_{3}$ is $3.7 \mathrm{~cm}$ and $2.14 \mathrm{~cm}$ in $\mathrm{SiC}$ with the penetration depth of $81.8 \mathrm{~cm}$ in $\mathrm{Al}_{2} \mathrm{O}_{3}$ and $0.8 \mathrm{~cm}$ in $\mathrm{SiC}$. Since the dielectric properties are temperature-independent, the absorbed microwave power remains constant during heating.

Uniform temperatures are observed in $\mathrm{Al}_{2} \mathrm{O}_{3}$, though a small peak in temperature is observed at the center of the $\mathrm{SiC}$ sample. The high thermal conductivities (low $B i_{c}$ numbers) of $\mathrm{Al}_{2} \mathrm{O}_{3}$ and $\mathrm{SiC}$ explain the constant temperature profile within the samples. Due to the greater dielectric loss of $\mathrm{SiC}$ (27.99) over $\mathrm{Al}_{2} \mathrm{O}_{3}(0.1566)$, the power absorbed in $\mathrm{SiC}$ is an order of magnitude greater, resulting in higher final temperatures. The high penetration depth in $\mathrm{Al}_{2} \mathrm{O}_{3}$ causes virtually no attenuation in the sample and standing wave patterns give rise to a power peak in the center of the sample. In contrast, due to the small penetration depth of $0.8 \mathrm{~cm}$ in $\mathrm{SiC}$, the power decays rapidly from the faces. The property of $\mathrm{SiC}$ to rapidly heat in the presence of microwaves is exploited to
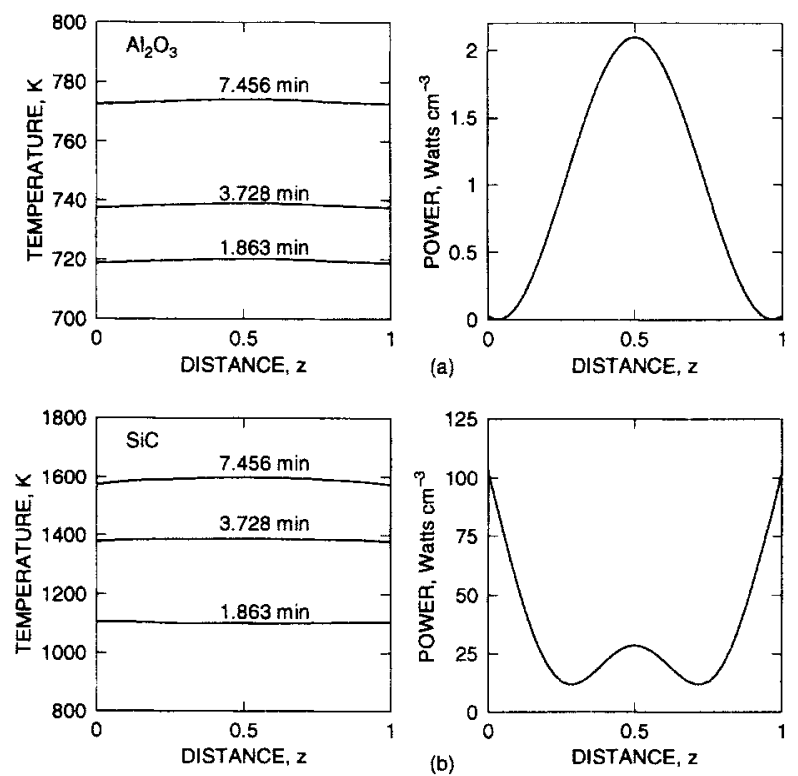

Figure 2. Power and temperature distributions for a 2 $\mathrm{cm}$ slab of (a) $\mathrm{Al}_{2} \mathrm{O}_{3}$ and (b) $\mathrm{SiC}$ exposed to microwaves from both sides.

$f=2,450 \mathrm{MHz}$, incident microwave intensity of $70 \mathrm{~W} \cdot \mathrm{cm}^{-2}$.
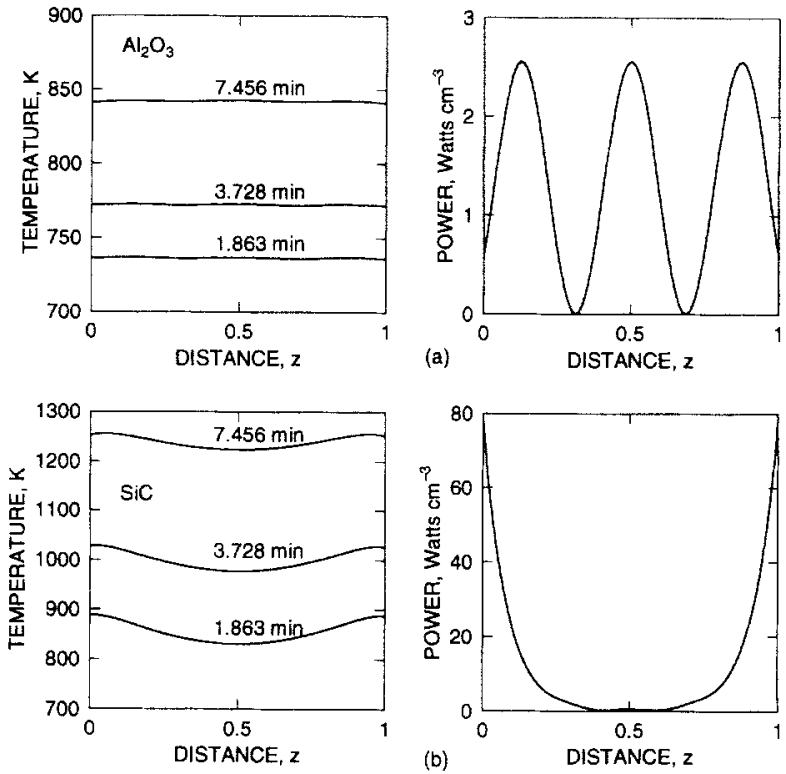

Figure 3. Power and temperature distributions for a 5 cm slab of (a) $\mathrm{Al}_{2} \mathrm{O}_{3}$ and (b) $\mathrm{SiC}$ exposed to microwaves from both sides.

$f=2,450 \mathrm{MHz}$, incident microwave intensity of $70 \mathrm{~W} \cdot \mathrm{cm}^{-2}$.

indirectly heat low loss ceramics like $\mathrm{Al}_{2} \mathrm{O}_{3}$, with $\mathrm{SiC}$ being used as a susceptor or lining causing heat to be transferred by radiation to a low loss sample such as $\mathrm{Al}_{2} \mathrm{O}_{3}$.

To investigate the effect of slab thickness on the power absorption and temperatures, we studied the heating of 5 and $10 \mathrm{~cm}$ thick samples (not shown) under the same conditions as the $2-\mathrm{cm}$ slab. Though the temperature profile in the $5 \mathrm{~cm}$ $\mathrm{Al}_{2} \mathrm{O}_{3}$ sample (Figure 3) is qualitatively similar to the $2 \mathrm{~cm}$ sample, the absorbed power has 3 peaks within the $5 \mathrm{~cm}$ sample. In contrast, the maxima of the absorbed power occurs at the center of the $2 \mathrm{~cm}$ sample. The absorbed power within the $5 \mathrm{~cm}$ SiC sample follows Lambert's law decaying exponentially into the sample. As a consequence, the temperature profiles have a minimum at the center, and the surfaces of the $5 \mathrm{~cm} \mathrm{SiC}$ sample heat up rapidly.

It should be noted that the low Biot numbers result in uniform temperatures for the $\mathrm{Al}_{2} \mathrm{O}_{3}$ sample; however, the large variation in power in the $\mathrm{SiC}$ samples leads to a $30^{\circ}$ variation in the $5 \mathrm{~cm}$ slab (Figure 3) and about $50^{\circ}$ in the $10 \mathrm{~cm}$ slab. Although a lumped parameter model based on an average power distribution for the temperature could be used for a low dielectric loss (high penetration depth) material like $\mathrm{Al}_{2} \mathrm{O}_{3}$, this would be less appropriate for a high dielectric loss (low penetration depth) material like SiC where the spatial power variation is large. In the next section we use a lumped parameter heating model to investigate the steady states in an $\mathrm{Al}_{2} \mathrm{O}_{3}$ sample.

\section{Microwave heating of $\mathrm{Al}_{2} \mathrm{O}_{3}$ with temperature-dependent dielectric properties}

The dielectric properties of $\mathrm{Al}_{2} \mathrm{O}_{3}$ as a function of temperature are tabulated in Table 2. Cubic splines were used to fit the dielectric data with temperature as shown in the inset of Figure 4a. Within this temperature range, we have investi- 
Table 2. Dielectric Data for $\mathrm{Al}_{2} \mathrm{O}_{3}$ $\left(\right.$ Density $=3.76 \mathrm{~g} \cdot \mathrm{cm}^{-3}$ ) at $2.45 \mathrm{GHz}^{*}$

\begin{tabular}{rll}
\hline${ }^{\circ} \mathrm{C}$ & \multicolumn{1}{c}{$\kappa^{\prime}$} & $\kappa^{\prime \prime}$ \\
\hline 296 & 9.46 & 0.01 \\
491 & 9.82 & 0.025 \\
683 & 10.15 & 0.055 \\
871 & 10.4 & 0.093 \\
1,050 & 10.81 & 0.158 \\
1,221 & 11.18 & 0.241 \\
1,379 & 11.77 & 0.476 \\
\hline
\end{tabular}

*Dielectric data for $\mathrm{Al}_{2} \mathrm{O}_{3}$ obtained from Hutcheon et al. (1992).

gated the sensitivity of the steady states to different initial conditions by heating or cooling the sample. Figure 4 illustrates the average power $\bar{P}(\theta)$ and temperature profiles for a 5 -cm slab of $\mathrm{Al}_{2} \mathrm{O}_{3}$ exposed to microwaves from both faces. The inset in Figure $4 \mathrm{~b}$ is based on a lumped parameter heating model where the dimensionless heat generation (for a slab of thickness $2 L$ and $\left.T_{0}=700 \mathrm{~K}\right) H_{g}=4 \bar{P}(\theta) L^{2} / k_{\text {eff }} T_{0}$ and heat loss $H_{l}=2\left[B i_{c} \theta+B i_{r} F(\theta)\right]$ are plotted against $\theta$. The unique steady state of $1,000 \mathrm{~K}$ obtained for both heating and cooling is similar to that predicted by the lumped parameter model when $H_{\mathrm{g}}=H_{l}$.
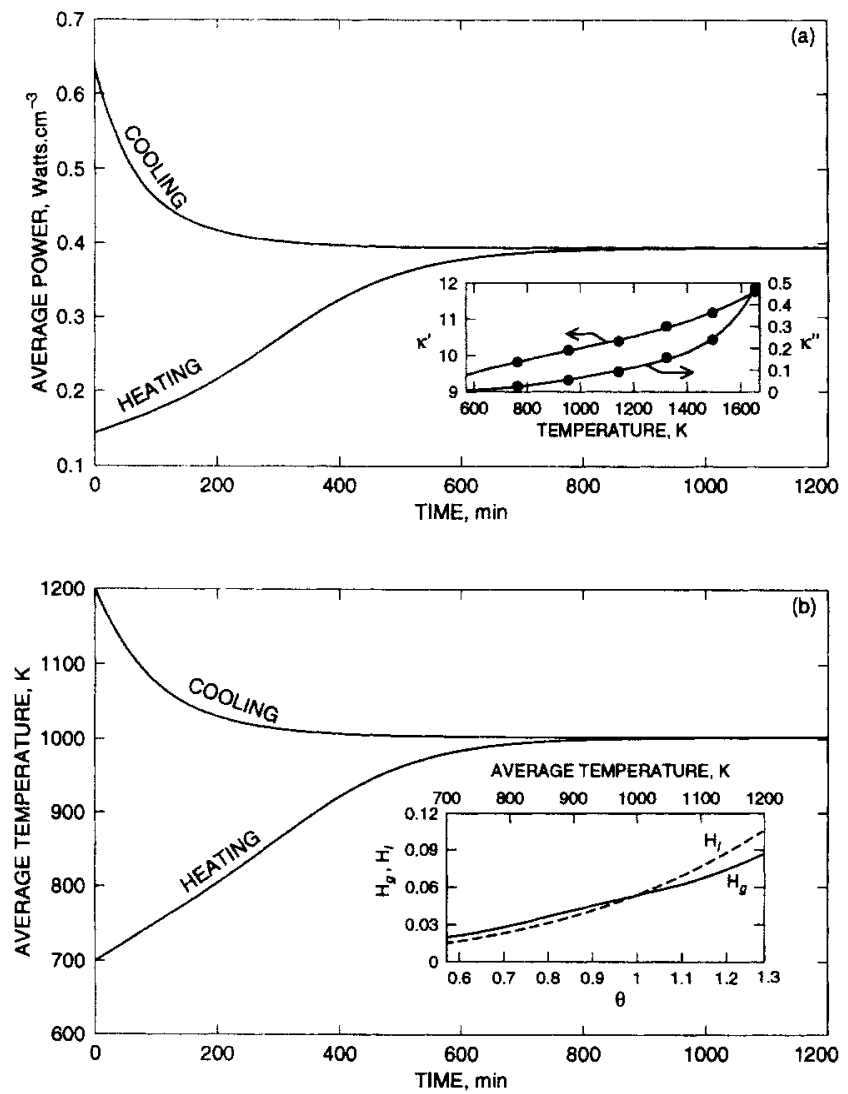

Figure 4. Average power and average temperature distributions for a $5^{\circ} \mathrm{cm}$ sample of $\mathrm{Al}_{2} \mathrm{O}_{3}$ with temperature-dependent dielectric properties exposed to microwaves from both sides.

$f=2,450 \mathrm{MHz}$, incident microwave intensity of $40 \mathrm{~W} \cdot \mathrm{cm}^{-2}$.

\section{Conventional sintering}

We have solved the evolution equations (Eqs. 20 and 21) using the Runge-Kutta method to study the influence of temperature on densification and grain growth. In these calculations we have assumed only heat loss by convection. Results are illustrated with respect to a normalized grain diameter $\bar{G}=G / x_{0}$, where $x_{0}=0.085 \mu \mathrm{m}$. The parameters used are similar to those used by Rödel and Glaeser (1990) in their experiments with $\mathrm{Al}_{2} \mathrm{O}_{3}$ crystals, where: $\psi=60^{\circ}, \Omega=2.11 \times$ $10^{-29} \mathrm{~m}^{3}, \delta D_{s}=3 \times 10^{-21} \mathrm{~m}^{3} \cdot \mathrm{s}^{-1}, M_{b}=5 \times 10^{-16} \mathrm{~m}^{3} \cdot \mathrm{N}^{-1}$. $\mathrm{s}^{-1}, \gamma_{s}=0.9 \mathrm{~J} \cdot \mathrm{m}^{-2}, \gamma_{b}=2 \gamma_{s} \cos \psi, \beta_{1}=0.6, \eta=1.05, \alpha_{1}=$ 1.0 , and $\delta D_{b} / \delta D_{s}=0.1$.

Figure 5a shows densification $\rho=1-\phi$ vs. time and Figure 5 b illustrates the normalized grain diameter $\bar{G}$ vs. $\rho$ for samples sintered at two different temperatures $1,473 \mathrm{~K}$ and $1,673 \mathrm{~K}$. We start with an initial porosity of $\phi=0.15$, and an initial grain size $G_{0}=1.43 \mu \mathrm{m}$ with zero hydrostatic stress $\left(\sigma_{h}=0\right)$. It is seen that densification is slower at higher temperatures. Figure $5 \mathrm{~b}$ illustrates the larger grain growth that occurs at a higher temperature, indicating that the time taken for densification is greater at higher temperatures allowing greater grain growth. The evolution equations (Eqs. 20 and 21) show that the porosity change during the late stages of sintering is inversely proportional to the temperature. Due to larger grains and longer diffusion paths along the grain boundaries, the rate of densification slows down during the late stages of sintering. It can be concluded that higher temperatures retard densification during the late stages of sintering. The same is true for a higher heating rate. Figures $5 \mathrm{c}$ and $5 \mathrm{~d}(T=1,673 \mathrm{~K})$ illustrate the enhanced densification and grain growth as the grain boundary diffusion coefficient $\delta D_{b}$ is increased.

\section{Microwave sintering of ceramics}

The microwave sintering of $\mathrm{Al}_{2} \mathrm{O}_{3}$ is analyzed by solving the electric field equations coupled with the energy balance equation (Eq. 15), and evolution equations for porosity and grain diameter (Eqs. 20 and 21) during the late stages of sintering. The effective dielectric and thermal properties of medium are functions only of the changing porosity $\phi$. The thermal properties of $\mathrm{Al}_{2} \mathrm{O}_{3}$ and air are tabulated in Table 1. Thermal and dielectric properties were assumed to be independent of temperature and the dielectric properties of $\mathrm{Al}_{2} \mathrm{O}_{3}$ used were $\kappa^{\prime}=11.77$ and $\kappa^{\prime \prime}=0.476$. The frequency of radiation is $2.45 \mathrm{GHz}$ flux of the incident power $30 \mathrm{~W}$. $\mathrm{cm}^{-2}$, and the ambient temperature $300 \mathrm{~K}$. Experimental sintering temperatures for $\mathrm{Al}_{2} \mathrm{O}_{3}$ are around $1,773 \mathrm{~K}$ or above for the intermediate and late stages of sintering. The dependence of grain boundary diffusion coefficient and surface diffusion coefficient on temperature is not known a priori and constant values for the diffusion coefficients were assumed. The grain. boundary diffusion coefficient $\delta D_{b}=3.0 \times 10^{-22}$ $\mathrm{m}^{3} \cdot \mathrm{s}^{-1}$ and all other parameters for $\mathrm{Al}_{2} \mathrm{O}_{3}$ are similar to those used for studying conventional sintering. Figure 6 illustrates the temperature, power, densification and grain diameter variation with time for a $1-\mathrm{cm}$ slab of $\mathrm{Al}_{2} \mathrm{O}_{3}$ during microwave sintering. An uniform initial porosity of 0.15 and grain diameter of $1.43 \mu \mathrm{m}$ was assumed.

The microwave power absorption profile shows that there is almost no loss, because the sample is small compared to 

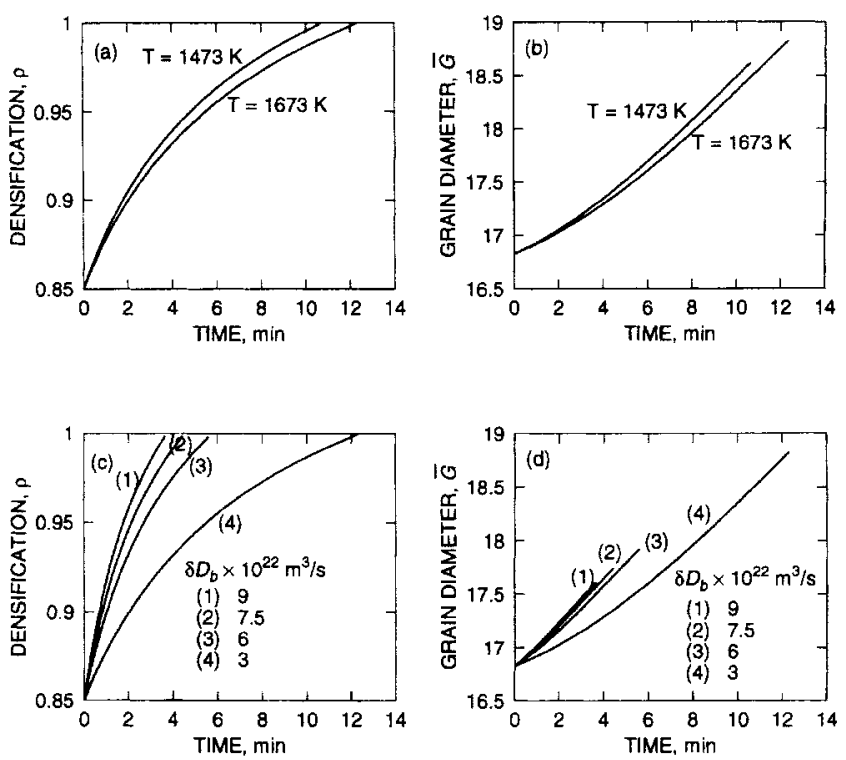

Figure 5. Evolution of (a) densification and (b) grain size as a function of time for two different temperatures, and (c) densification and (d) grain diameter as a function of time for various grain boundary diffusion coefficients.

the penetration depth $(\sim 28 \mathrm{~cm})$ in the sample. The average power absorbed in the sample after $1 \mathrm{~min}$ of heating is around $2 \mathrm{~W} \cdot \mathrm{cm}^{-3}$, the corresponding temperature is $1,720 \mathrm{~K}$, and densification is 0.88 . Since the dielectric loss is a function of the changing porosity in the medium, the power absorbed in
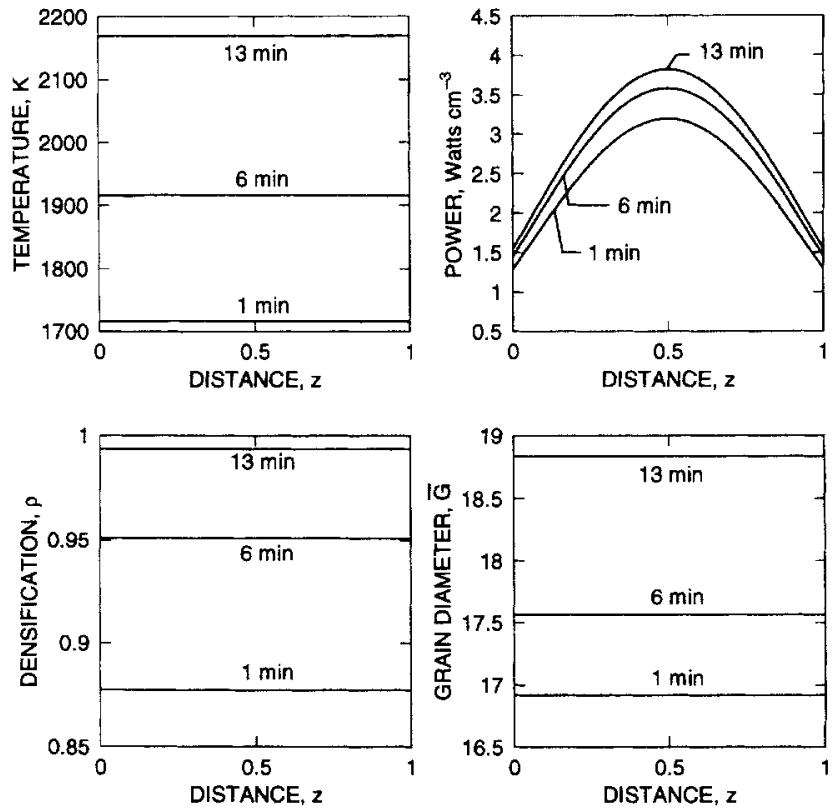

Figure 6. Temperature, power, densification, and grain size profiles for a $1 \mathrm{~cm}$ slab of $\mathrm{Al}_{2} \mathrm{O}_{3}$ being sintered by microwaves of equal intensity from both sides.

$f=2,450 \mathrm{MHz}$, incident microwave intensity of $30 \mathrm{~W} \cdot \mathrm{cm}^{-2}$.
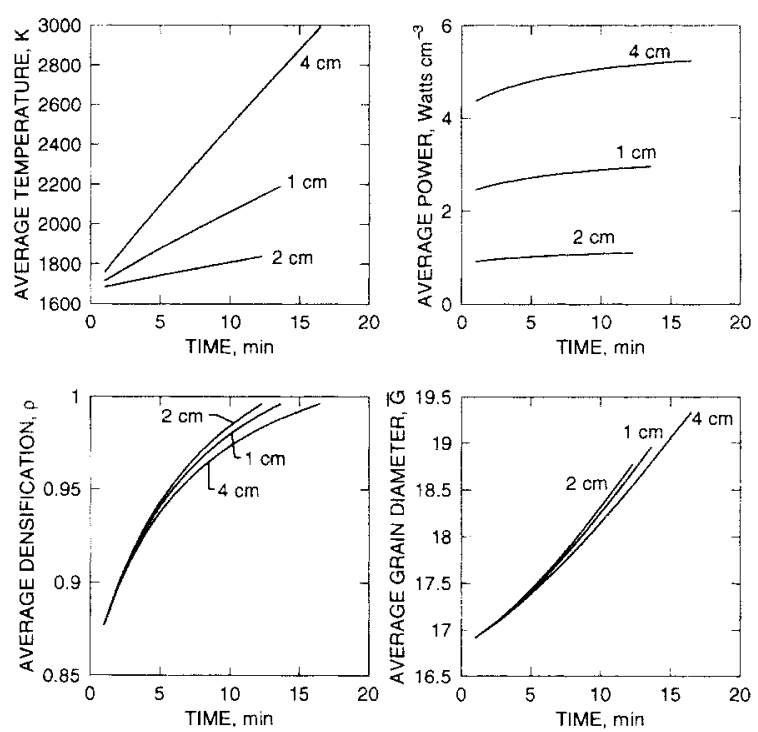

Figure 7. Average temperature, power, densification and grain size profiles for different slabs of $\mathrm{Al}_{2} \mathrm{O}_{3}$ being sintered by microwaves of equal intensity from both sides.

$f=2,450 \mathrm{MHz}$, incident microwave intensity of $30 \mathrm{~W} \cdot \mathrm{cm}^{-2}$

the sample changes with time. Like conventional sintering, the densification, which is spatially uniform, increases with temperature. The time taken for reaching a density of 0.996 was $13.21 \mathrm{~min}$, and the final temperature of the sample was $2,180 \mathrm{~K}$. The size of the grain at the end of sintering was 1.58 $\mu \mathrm{m}$.

Figure 7 illustrates the variation of average temperature, power, densification and normalized grain diameter with time for the different slab thicknesses. Average values were calculated by integrating the variable across the sample length. The final densification is 0.996 for all the sample lengths. A higher temperature is observed for the $4 \mathrm{~cm}$ sample whereas the lowest temperature is observed for the $2 \mathrm{~cm}$ sample. Although the average power absorption with time shows a small deviation from linearity, the average temperatures vary linearly with time. The maximum average grain size occurs in the sample which has taken the longest time to densify. This is consistent with the trends observed while analyzing conventional sintering, where high temperatures result in greater grain growth, increasing the densification time.

The variation in heating rates for the different slabs can be explained by examining the average microwave power absorbed as a function of slab thickness (Figure 8). Ayappa (1997) showed that resonances are a function of the sample dimension and wavelength of radiation $\lambda_{m}$ in the medium. During a resonance, the average power absorbed exhibits a local maximum. The study showed that for slabs of thickness $L$ and integer values of $n$ resonances were found to occur at $L / \lambda_{m}=0.5 n$ for microwave's incident from one face, and for incidence from both faces, resonances occur at $L / \lambda_{m}=n$. The first resonance is found to occur at a slab thickness of $3.7 \mathrm{~cm}$, which is the wavelength in the medium. The resonance at 3.7 $\mathrm{cm}$ leads to the higher heating rate for the $4-\mathrm{cm} \mathrm{slab}$, and destructive interference leads to a lower heating rate at $1 \mathrm{~cm}$. 


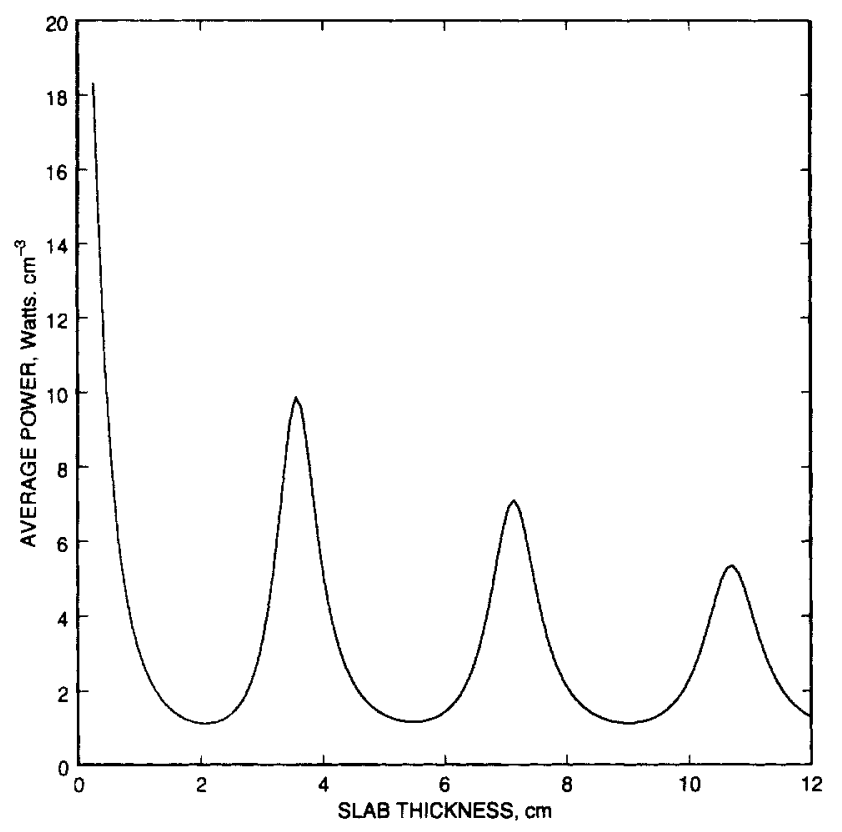

Figure 8. Resonance in slabs exposed to microwaves from both sides as a function of the length of the slab.

$f=2,450 \mathrm{MHz}$, incident microwave intensity of $30 \mathrm{~W} \cdot \mathrm{cm}^{-2}$.

\section{Comparison of microwave and conventional sintering}

One of our primary objectives was to compare the model for microwave sintering with the sintering model in the absence of microwaves. A meaningful comparison of the densification and grain growth between conventional and microwave sintering can be made only if both sintering processes are carried out at similar heating rates. Experimentally, this is difficult to achieve since controlling the temperature rise during microwave sintering is not a simple issue. This is primarily due to the rapid increase in dielectric loss with temperature.

Here we compare conventional sintering and microwave sintering in the following manner. The initial temperature, densification, and the grain size distribution for both conventional and microwave heating were the same. In order to facilitate a fair comparison between the conventional and microwave sintering models we have used systems with low Biot numbers, where an average temperature and microwave heating rate can be obtained and input into the grain boundary and pore evolution equations. From our microwave sintering study, we observe that the average temperature follows a linear relationship with time for all the samples studied (Figure 7). We calculate the microwave heating rate, based on a linear temperature-time relationship of the form $T=a$ $+b t$, where $a$ is the initial temperature. The average heating rate for the $1 \mathrm{~cm}$ sample is around $37.4^{\circ} / \mathrm{min}$. This heating rate is used in the model for conventional sintering, and the sample is allowed to heat for the same time as in the case of microwave heating. In Figure 9 the average densification and normalized grain size from the microwave sintering simulation has been plotted against the densification and grain sizes obtained from the conventional sintering model. It is seen
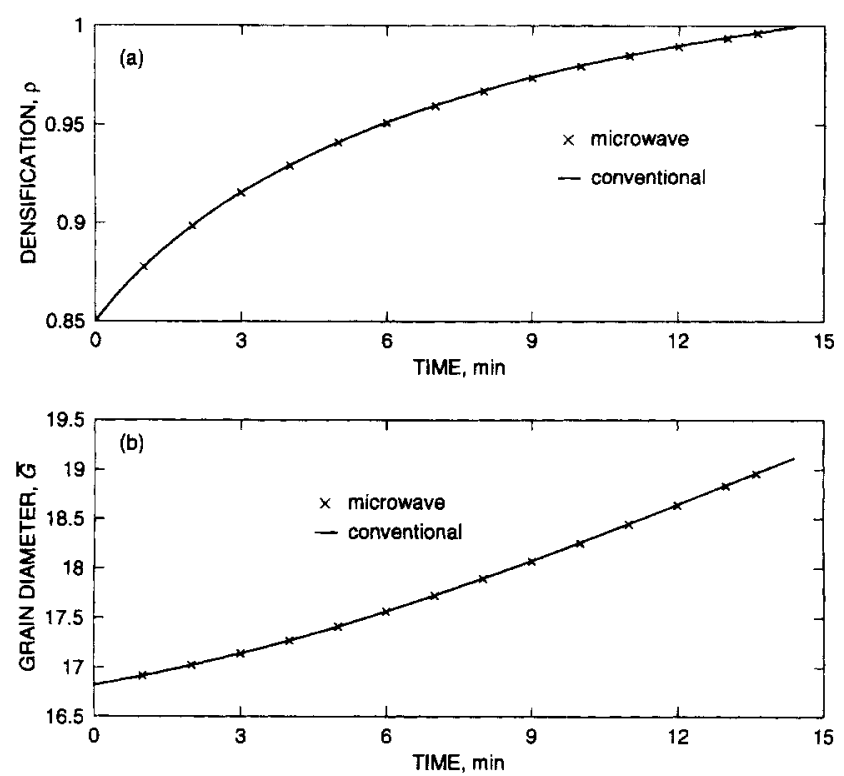

Figure 9. Comparison of densification and grain size profiles for a $1 \mathrm{~cm}$ slab of $\mathrm{Al}_{2} \mathrm{O}_{3}$ by $\mathrm{mi}$ crowave and conventional heating for the same heating rate.

that the profiles are identical for both sintering methods. A similar result is observed at other slab thicknesses as well. We can conclude that during the late stages of sintering, the models for microwave and conventional sintering predict similar sintering characteristics.

The experimental results of Janney and Kimrey (1988), which show that microwaves enhance densification rates during the early stages of sintering, cannot be illustrated by our model since the evolution equations are valid only for the late stages of sintering. To model the early stages of sintering, specific evolution equations for the grain size and porosity have to be developed. One of the mechanisms proposed to explain enhanced densification during microwave sintering is based on the assumption that grain boundary diffusion coefficients might be higher in the presence of microwaves. Whether this occurs during microwave sintering is open to question. It must be noted here that our model for microwave sintering incorporates the effect of microwaves only as a volumetric source of heat. If the microwaves have an effect, other than thermal, on the transport properties like the grain boundary diffusion coefficient, then a model for microwave sintering must incorporate these effects as well.

\section{Conclusion}

In an effort to analyze the influence of microwaves during sintering we have developed a model which incorporates the electric field equations and the heat conduction equation along with the evolution equations for porosity and grain diameter (Svoboda and Riedel, 1992) to predict temperature, power, densification, and grain size distributions during microwave sintering. The coupled equations are solved using a Galerkin finite-element method. 
Heating in slabs of $\mathrm{Al}_{2} \mathrm{O}_{3}$ and $\mathrm{SiC}$ in the absence of sintering illustrates the higher heating rates that are observed in $\mathrm{SiC}$ due to its greater dielectric loss. The low Biot numbers in these ceramics result in spatially uniform temperature distributions. The uniformity in temperatures for $\mathrm{Al}_{2} \mathrm{O}_{3}$ and the lumped parameter heating model for steady states suggest that a lumped parameter model based on an average power would be sufficient to predict temperatures. However, in samples like $\mathrm{SiC}$ where the penetration depths are small, this may not be appropriate. Heating and cooling studies for $\mathrm{Al}_{2} \mathrm{O}_{3}$ with temperature-dependent dielectric properties resulted in a unique steady state.

Using the model of Svoboda and Riedel (1992), we studied the effect of temperature and grain boundary diffusion coefficient during the late sintering of $\mathrm{Al}_{2} \mathrm{O}_{3}$. An increase in temperature lowers the rate of densification. Densification rates are higher for larger grain boundary diffusion coefficients since the model assumes that pores shrink only by grain boundary diffusion.

Microwave sintering studies for $\mathrm{Al}_{2} \mathrm{O}_{3}$ slabs with the same incident power show that samples with a higher heating rate take a longer time to sinter. This is in agreement with the conventional sintering studies. Since the penetration depth in $\mathrm{Al}_{2} \mathrm{O}_{3}$ is quite large, we find that heating rates are a strong function of the slab thickness due to resonance effects. In all cases a linear heating rate was observed during MW sintering.

Perhaps one of the central issues that has driven research in microwave sintering is the observed increase in densification when compared with conventional oven sintering. Using the heating rates obtained from the microwave sintering calculations, we solved the evolution equations for porosity and grain diameter in the absence of microwaves. We find that the time taken for sintering, densification rates, and grain growths are identical for both conventional and microwave sintering. Within the framework of our model, this indicates that the effect of microwaves is purely thermal. Although an increase in grain boundary diffusion coefficient in the sintering model is seen to enhance densification, the enhanced densification observed in MW sintering experiments cannot be predicted unless a specific mechanism or model is developed to predict such an increase in the presence of MWs.

\section{Acknowledgment}

We thank the Supercomputer Education and Research Center, Indian Institute of Science for computing resources, as well as Vikram Jayaram, Atul Chokshi and K. J. Rao for useful discussions on sintering and and Madhuchhanda Bhattacharya for assistance in computations.

\section{Literature Cited}

Ayappa, K. G., H. T. Davis, E. A. Davis, and J. Gordon, "Analysis of Microwave Heating of Materials with Temperature-Dependent Properties," AIChE J., 37, 313 (1991).

Ayappa, K. G., "Modelling Transport Processes During Microwave Heating: A Review," Rev. in Chem. Eng., 13, 1 (1997).

Ceramic Source, compilted by American Ceramic Society, Vol. 7, (1991-1992).

Hutcheon, R. M., M. S. De Jong, F. P. Adams, P. G. Lucuta, J. E. McGregor, and L. Bahen, "RF and Microwave Dielectric Mea- surements to $1400^{\circ} \mathrm{C}$ and Dielectric Loss Mechanisms," Mater. Res. Soc. Symp. Proc., 269, 541 (1992).

Janney, M. A., and H. D. Kimrey, "Microwave Sintering of Alumina at $28 \mathrm{GHz}$, " Ceramic Powder Sci., 1, 919 (1988).

Johnson, D. L., "Microwave Heating of Grain Boundaries in Ceramics," J. Am. Ceram. Soc., 74, 849 (1991).

Kingery, W. D., H. K. Bowen, and D. R. UhImann, An Introduction to Ceramics, Wiley, New York (1991).

Kriegsmann, G. A., M. E. Brodwin, and D. G. Watters, "Microwave Heating of a Ceramic Halfspace," SLAM J. Appl. Math., 50, 1088 (1990).

Meek, T. T., "Proposed Model for the Sintering of a Dielectric in a Microwave Field," J. Mater. Sci. Lett., 6, 638 (1987).

Rao, K. J., and P. D. Ramesh, "Use of Microwaves for the Synthesis and Processing of Materials," Bull. Mater. Sci., 18, 447 (1995).

Rödel, J., and A. M. Glaeser, "Pore Drag and Pore-Boundary Separation in Alumina," J. Am. Ceram. Soc., 73, 3302 (1990).

Rothman, S. J., "Critical Assessment of Microwave-Enhanced Diffusion," Mat. Res. Soc. Symp. Proc., 347, 9 (1994).

Samuels, J., and J. R. Brandon, "Effect of Composition on the Enhanced Microwave Sintering of Alumina-Based Ceramic Composites," J. Mater. Sci., 27, 3259 (1992).

Sutton, W. H., "Microwave Processing of Ceramic Materials," Am. Ceram. Soc. Bull., 68, 376 (1989).

Svoboda, J., and H. Riedel, "Pore-Boundary Interactions and Evolution Equations for the Porosity and the Grain Size During Sintering," Acta Metall., 40, 2829 (1992).

Thomas, Jr., J. R., J. D. Katz, and R. D. Blake, "Temperature Distribution in Microwave Sintering of Alumina Cylinders," Mat. Res. Soc. Symp. Proc., 347, 311 (1994).

Welty, J. R., C. E. Wicks, and R. E. Wilson, Fundamental of Momentum, Heat and Mass Transfer, Wiley, New York (1976).

Xie, Z., J. Li, Y. Huang, and X. Kong, "Microwave Sintering Behaviour of $\mathrm{ZrO}_{2}-\mathrm{Y}_{2} \mathrm{O}_{3}$ with Agglomerate," J. Mater. Sci. Lett., 15, 1158 (1996).

\section{Appendix}

\section{Finite-element analysis}

For a general equation of the form

$$
L u=f
$$

Galerkin finite elements consist of expanding the unknown $u$ in a finite-element basis set $\Phi$. Thus

$$
u \approx \tilde{u}=\sum_{j=1}^{N} y_{j} \Phi_{j}(z) .
$$

The error $L \vec{u}-f$ is set orthogonal to the basis functions, and

$$
\int_{0}^{1}(L \tilde{u}-f) \Phi_{i} d z=0
$$

for

$$
i=1, \ldots, N
$$

Integrating by parts, and incorporating boundary and interface conditions, Eq. A3 results in a set of algebraic equations whose solution yields the unknown coefficients $y_{j}$ of the expansion. 
Expanding the real $\left(v_{x}\right)$ and imaginary components $\left(w_{x}\right)$ of the electric field, the temperature $(\theta)$, the porosity $(\phi)$, and the normalized grain diameter $(\bar{G})$ in the basis $\{\Phi\}$,

$$
\begin{array}{cc}
\tilde{v}_{x}=\sum_{k=1}^{N} v_{k} \Phi_{k}(z), & \tilde{w}_{x}=\sum_{k=1}^{N} w_{k} \Phi_{k}(z), \\
\tilde{\theta}=\sum_{k=1}^{N} \theta_{k}\left(\tau_{1}\right) \Phi_{k}(z), & \tilde{\phi}=\sum_{k=1}^{N} \phi_{k}\left(\tau_{2}\right) \Phi_{k}(z),
\end{array}
$$

and

$$
\tilde{\bar{G}}=\sum_{k=1}^{N} \overline{G_{k}}\left(\tau_{2}\right) \Phi_{k}(z)
$$

for

$$
0 \leq z \leq 1
$$

The Galerkin finite-element method yields the following nonlinear residual equations for Eqs. 11, 12, 16, 20, and 21, respectively.

$$
\begin{array}{r}
R_{i}^{(1)}=\sum_{k=1}^{N} v_{k}^{t+1} \int_{0}^{1} \Phi_{i}^{\prime} \Phi_{k}^{\prime} d z-\sum_{k=1}^{N} v_{k}^{t+1} \int_{0}^{1} \psi\left(\phi^{t+1}\right) \Phi_{i} \Phi_{k} d z \\
+\sum_{k=1}^{N} w_{k}^{t+1} \int_{0}^{1} \chi\left(\phi^{t+1}\right) \Phi_{i} \Phi_{k} d z+\frac{2 \omega L}{c} w_{1}^{t+1} \delta_{i 1} \\
+\frac{4 \omega L}{c} \sin (\omega L / c) \delta_{i 1}+\frac{2 \omega L}{c} w_{N}^{t+1} \delta_{i N} \\
+\frac{E_{R}}{E_{L}} \frac{4 \omega L}{c} \sin (\omega L / c) \delta_{i N},
\end{array}
$$

$$
\begin{aligned}
R_{i}^{(2)}= & \sum_{k=1}^{N} w_{k}^{t+1} \int_{0}^{1} \Phi_{i}^{\prime} \Phi_{k}^{\prime} d z-\sum_{k=1}^{N} v_{k}^{t+1} \int_{0}^{1} \chi\left(\phi^{t+1}\right) \Phi_{i} \Phi_{k} d z \\
& -\sum_{k=1}^{N} w_{k}^{t+1} \int_{0}^{1} \psi\left(\phi^{t+1}\right) \Phi_{i} \Phi_{k} d z-\frac{2 \omega L}{c} v_{1}^{t+1} \delta_{i 1} \\
& +\frac{4 \omega L}{c} \cos (\omega L / c) \delta_{i 1}-\frac{2 \omega L}{c} v_{N}^{t+1} \delta_{i N} \\
& +\frac{E_{R}}{E_{L}} \frac{4 \omega L}{c} \cos (\omega L / c) \delta_{i N},
\end{aligned}
$$

$$
\begin{aligned}
& R_{i}^{(3)}=\sum_{k=1}^{N} \int_{0}^{1}\left(\overline{\rho C_{p}}\right)^{t+1}\left\{\frac{\theta_{k}^{t+1}-\theta_{k}^{t}}{\Delta \tau_{1}}\right\} \Phi_{i} \Phi_{k} d z \\
& +\sum_{k=1}^{N} \theta_{k}^{t+1} \int_{0}^{1} \bar{k}\left(\phi^{t+1}\right) \Phi_{i}^{\prime} \Phi_{k}^{\prime} d z-\frac{2 L^{2} \omega \epsilon_{0} E_{L}^{2}}{k_{0} T_{0}} \\
& \times \int_{0}^{1} \kappa^{\prime \prime}\left(\phi^{t+1}\right)\left\{\left(\sum_{k} v_{k}^{t+1} \Phi_{k}\right)^{2}+\left(\sum_{k} w_{k}^{t+1} \Phi_{k}\right)^{2}\right\} \\
& +\bar{k}_{1}\left(\phi^{t+1}\right)\left(B i_{c}\left(\phi^{t+1}\right) \theta_{1}^{t+1}+B i_{r} F\left(\theta_{1}^{t+1}\right)\right) \delta_{i 1} \\
& +\bar{k}_{N}\left(\phi^{t+1}\right)\left(B i_{c}\left(\phi^{t+1}\right) \theta_{N}^{t+1}+B i_{r} F\left(\theta_{N}^{t+1}\right)\right) \delta_{i N}, \\
& R_{i}^{(4)}=\sum_{k=1}^{N} \int_{0}^{1} \frac{\phi_{k}^{t+1}-\phi_{k}^{t}}{\Delta \tau_{2}} \Phi_{i} \Phi_{k} d z \\
& -\int_{0}^{1} \frac{24 \bar{Z} \Omega^{2} \gamma_{s} \delta D_{s} \delta D_{b}}{2\left(\gamma_{b} M_{b}\right)^{2} k^{2} T_{0}\left[\tilde{\theta} T_{0}+T_{\infty}\right] q(\bar{\omega})\left(\overline{\bar{G}} x_{0}\right)^{3}} \\
& \times\left[\sigma_{h}-\frac{2 \gamma_{s} \sin \psi}{\tilde{\bar{G}} x_{0}}\left(\frac{4 \bar{Z} h(\psi)}{\tilde{\phi}}\right)^{1 / 3}\right] \Phi_{i} d z,
\end{aligned}
$$

and

$$
\begin{aligned}
& R_{i}^{(5)}=\sum_{k=1}^{N} \int_{0}^{1} \frac{{\overline{G_{k}}}^{t+1}-{\overline{G_{k}}}^{t}}{\Delta \tau_{2}} \Phi_{i} \Phi_{k} d z-\int_{0}^{1} \frac{\alpha_{1}}{2 \overline{\bar{G}}} \\
& \times\left[1-\bar{\omega}+\frac{0.42 \eta M_{b} k\left(\tilde{\theta} T_{0}+T_{\infty}\right)\left(\tilde{\bar{G}} x_{0}\right)^{2}}{\beta_{1}^{2} p(\psi) \Omega \delta D_{s}}\left(\frac{\bar{\phi}}{\bar{Z} h(\psi)}\right)^{4 / 3}\right]^{-1} \Phi_{i} d z
\end{aligned}
$$

For a given value of the time increment $\Delta t$, corresponding values of $\Delta \tau_{1}$ and $\Delta \tau_{2}$ were used in Eqs. A7-A9. The residual equations (Eqs. A5-A9) result in a set of nonlinear algebraic equations that are solved using a Newton-Raphson scheme to determine the coefficients of the expansions in Eq. A4 at each time step and the microwave power is deduced from $\mathrm{Eq}$. 16. At each time step, the linear $(5 N \times 5 N)$ system solved is

$$
\boldsymbol{J}\left(\boldsymbol{u}^{n, t+1}\right)\left[\boldsymbol{u}^{n+1, t+1}-\boldsymbol{u}^{n, t+1}\right]=-\boldsymbol{R}\left(\boldsymbol{u}^{n, t+1}\right),
$$

where $n$ is the Newton iterate index, $t$ the time index, $J$ the Jacobian matrix, and $\boldsymbol{R}$ the vector of residuals.

Manuscript received Dec. 31, 1997, and revision received July 14, 1998. 